Results show a negative association between internet use and later bed times, earlier waking times, and sleep duration. Sleep quality was not related to internet use. Older adults' internet use may keep them awake while paradoxically not impacting psychological well-being.

\section{INTERPERSONAL RELATIONSHIP CHALLENGES AMONG STROKE SURVIVORS AND FAMILY CAREGIVERS}

M. McCarthy ${ }^{1}$, K. Lyons ${ }^{2}$, J. Schellinger ${ }^{3}$, K. Stapleton ${ }^{3}$, T. Bakas ${ }^{4}$, 1. College of Social and Behavioral Sciences Social Work, 2. Boston College, 3. University of Cincinnati, 4. University of Cincinnati - College of Nursing

A strong interpersonal relationship after stroke is important for the well-being of survivors and family caregivers. The aim of this study is to enhance understanding about factors that contribute to relationship challenges among stroke dyads. Semi-structured interviews were conducted with $\mathrm{N}=19$ spousal and non-spousal care dyads at approximately 3 month post-stroke. Qualitative data were analyzed using an interpretive description approach. Seven themes about factors that contribute to relationship challenges were identified, each with associated subthemes: (1) Coping with the direct effects of stroke; (2) Incongruence in perceptions between survivors and caregivers; (3) Lack of open communication; (4) Managing worries; (5) Adjusting to new roles; (6) Amplification of existing relationship issues, and; (7) Lack of support from other family members and friends. Findings highlight priority areas to consider in promoting strong relationships between survivors and family caregivers. Future research is needed to design interventions that family practitioners can use to disrupt negative communication cycles, promote empathy and collaboration between partners, and help dyads achieve a balance so that each person's needs are met.

\section{INTERPERSONAL TENSIONS AND PAIN AMONG OLDER ADULTS: THE MEDIATING ROLE OF NEGATIVE MOOD}

J. Graham ${ }^{1}$, M. Huo ${ }^{1}$, K. Birditt 2 , S. Charles ${ }^{3}$, K. Fingerman ${ }^{1}, 1$. The University of Texas at Austin, 2. The University of Michigan, 3. The University of California, Irvine

Pain is common in late life and may be exacerbated when older adults incur negative social encounters. Although negative emotion may explain this link, few studies have directly examined this hypothesis. Further, closeness may matter in regard to these associations. This study examined (a) the link between negative social encounters and pain throughout the day, (b) whether the link is mediated by negative emotion, and (c) whether the links vary by closeness with social partners. Older adults $(n=313)$ completed ecological momentary assessments and reported their social encounters and experiences of pain every 3 hours throughout the day for 5 to 6 days. Multilevel models revealed that negative encounters with any social partner was associated with increased pain at each 3-hour interval. Further, the models revealed evidence of full mediation such that negative social encounters led to pain due to increased negative mood. Additionally, negative encounters with less close social partners were associated with pain; yet, negative encounters with close social partners were not associated with pain. This work suggests that emotion regulation in the context of negative social encounters may be a key factor to improve the daily lives of older adults in pain.

\section{INTERPROFESSIONAL SPIRITUAL CARE TRAINING FOR GERIATRIC CARE PROVIDERS}

J. Bandini' ${ }^{1}$, M. Thiel ${ }^{2}$, E. Meyer ${ }^{3}$, S. Paasche-Orlow ${ }^{2}$, Q. Zhang ${ }^{1}$, W. Cadge ${ }^{1}$, 1. Brandeis University, 2. Hebrew SeniorLife, 3. Boston Children's Hospital

Religion and spirituality have been shown to provide older patients and their families with a source of strength, hope, coping, and a sense of meaning in their life (Koenig et al. 1997; Krause et al. 2003). Despite the importance patients place in religion and spirituality, many patients with advanced diseases report that their religious and spiritual needs are not met by their health care team, and many nonchaplain clinicians feel unprepared to address religious and spiritual issues in their clinical practice (Balboni et al. 2013; Puchalski 2012). The purpose of this study was to assess the efficacy of a one-day workshop on spiritual care for nonchaplain clinicians who provide care elderly long-term care patients. Clinician participants $(\mathrm{N}=68)$ were given a pre-survey at the beginning of the workshop, a post-survey at the conclusion of the workshop, and a three month follow-up survey to evaluate their comfort in engaging in spiritual issues with patients and families before and after the workshop. Overall scores for clinicians' self-reported perceived ability and comfort in engaging in issues around spirituality with patients and their families increased significantly right after the training and after three months, although this improvement slightly diminished after three months compared to at the end of the day of the spiritual generalist workshop. This study suggests that a spiritual care training program targeted towards geriatric clinicians has the potential to provide clinicians with the tools, skills, and support they need to approach basic spiritual care with their patients and family members.

\section{INTERVENTION COMPARATIVE EFFECTIVENESS FOR ADULT COGNITIVE TRAINING (ICE-ACT) (CLINICALTRIALS\#:NCT03141281)}

J. Yoon ${ }^{1}$, N. Roque ${ }^{2}$, R. Andringa ${ }^{1}$, K. Griffis Lewis ${ }^{3}$, E. Harrell ${ }^{1}$, T. Vitale ${ }^{1}$, N. Charness ${ }^{1}, 1$. Florida State University, 2. Penn State University, 3. Department of Psychology, Florida State University

Age-related perceptual and cognitive declines are associated with difficulties performing everyday tasks required to remain independent. Encouraging improvements in cognitive abilities have been shown for various short-term interventions (e.g., the ACTIVE trial, digital games, aerobic exercise) but there is little evidence for direct impact on independence. This project compared the effect of broad and directed (narrow) technology-based training on basic perceptual and cognitive abilities in older adults and on the performance of simulated tasks of daily living including driving and fraud avoidance. Participants $(\mathrm{N}=230$, Mean age $=72)$ were randomly assigned to four training conditions: broad training using either 1) a web-based brain game or 2) the video game, or to directed training for 3) Instrumental Activities of Daily Living (IADL) training using web-based programs for both 